\title{
Business forecasting methods
}

\author{
Rob J Hyndman
}

November 8, 2009

\section{Forecasting, planning and goals}

Forecasting is a common statistical task in business, where it helps inform decisions about scheduling of production, transportation and personnel, and provides a guide to long-term strategic planning. However, business forecasting is often done poorly and is frequently confused with planning and goals. They are three different things.

Forecasting is about predicting the future as accurately as possible, given all the information available including historical data and knowledge of any future events that might impact the forecasts.

Goals are what you would like to happen. Goals should be linked to forecasts and plans, but this does not always occur. Too often, goals are set without any plan for how to achieve them, and no forecasts for whether they are realistic.

Planning is a response to forecasts and goals. Planning involves determining the appropriate actions that are required to make your forecasts match your goals.

Forecasting should be an integral part of the decision-making activities of management, as it can play an important role in many areas of a company. Modern organizations require short-, medium- and long-term forecasts, depending on the specific application.

Short-term forecasts are needed for scheduling of personnel, production and transportation. As part of the scheduling process, forecasts of demand are often also required.

Medium-term forecasts are needed to determine future resource requirements in order to purchase raw materials, hire personnel, or buy machinery and equipment.

Long-term forecasts are used in strategic planning. Such decisions must take account of market opportunities, environmental factors and internal resources.

An organization needs to develop a forecasting system involving several approaches to predicting uncertain events. Such forecasting systems require the development of expertise in identifying forecasting problems, applying a range of forecasting methods, selecting appropriate methods for each problem, and evaluating and refining forecasting methods over time. It is also important to have strong organizational support for the use of formal forecasting methods if they are to be used successfully.

\section{Commonly used methods}

Typically, businesses use relatively simple forecasting methods that are often not based on statistical modelling. However, the use of statistical forecasting is growing and some of the most commonly used methods are listed below. 


\subsection{Time series methods}

Let the historical time series data be denoted by $y_{1}, \ldots, y_{n}$, and the forecast of $y_{n+h}$ be given by $\hat{y}_{n+h \mid n}, h>0$.

- Naïve forecasting is where the forecast of all future values of a time series are set to be equal to the last observed value: $\hat{y}_{n+h \mid n}=y_{n}, h=1,2, \ldots$. If the data follow a random walk process $\left(y_{t}=y_{t-1}+e_{t}\right.$, where $e_{t}$ is white noise - a series of iid random variables with zero mean), then this is the optimal method of forecasting. Consequently, it is popular for stock price and stock index forecasting, and for other time series that measure the behaviour of a market that can be assumed to be efficient.

- Simple exponential smoothing was developed in the 1950s (Brown 1959) and has been widely used ever since. Forecasts can be computed recursively as each new data point is observed:

$$
\hat{y}_{t+1 \mid t}=\alpha y_{t}+(1-\alpha) \hat{y}_{t \mid t-1},
$$

where $0<\alpha<1$. (Longer-term forecasts are constant: $\hat{y}_{t+h \mid t}=\hat{y}_{t+1 \mid t}, h \geq 2$.) Consequently, only the most recent data point and most recent forecast need to be stored. This was an attractive feature of the method when computer storage was expensive. The method has proved remarkably robust to a wide range of time series, and is optimal for several processes including the $\operatorname{ARIMA}(0,1,1)$ process (Chatfield et al. 2001).

- Holt's linear method (Holt 1957) is an extension of simple exponential forecasting that allows a locally linear trend to be extrapolated. Forecasts are given by $\hat{y}_{t+h \mid t}=\ell_{t}+h b_{t}$, where

$$
\begin{aligned}
& \ell_{t}=\alpha y_{t}+(1-\alpha)\left(\ell_{t-1}+b_{t-1}\right), \\
& b_{t}=\beta\left(\ell_{t}-\ell_{t-1}\right)+(1-\beta) b_{t-1},
\end{aligned}
$$

and the two parameters $\alpha$ and $\beta$ must lie in $[0,1]$. Here $\ell_{t}$ denotes the level of the series and $b_{t}$ the slope of the trend at time $t$.

- For seasonal data, a popular method is the Holt-Winters method, also introduced in Holt (1957), which extends Holt's method to include seasonal terms. Then $\hat{y}_{t+h \mid t}=\ell_{t}+h b_{t}+$ $s_{t-m+h_{m}^{+}}$, where

$$
\begin{aligned}
& \ell_{t}=\alpha\left(y_{t}-s_{t-m}\right)+(1-\alpha)\left(\ell_{t-1}+b_{t-1}\right), \\
& b_{t}=\beta\left(\ell_{t}-\ell_{t-1}\right)+(1-\beta) b_{t-1}, \\
& s_{t}=\gamma\left(y_{t}-\ell_{t}\right)+(1-\gamma) s_{t-m},
\end{aligned}
$$

$h_{m}^{+}=[(h-1) \bmod m]+1$, and the three parameters $\alpha, \beta$ and $\gamma$ all lie in $[0,1]$.

There is also a multiplicative version of the Holt-Winters method, and damped trend versions of both Holt's linear method and the Holt-Winters method (Makridakis et al.|1998). None of these methods are explicitly based on underlying time series models, and as a result the estimation of parameters and the computation of prediction intervals is often not done. However, all the above methods have recently been shown to be optimal for some state space models (Hyndman et al. 2008), and maximum likelihood estimation of parameters, statistical model selection and computation of prediction intervals is now becoming more widespread.

Other time series models sometimes used in business forecasting include ARIMA models, GARCH models (especially in finance), structural models and neural networks. 


\subsection{Explanatory models for forecasting}

The use of explanatory models in business forecasting does not have such a long history as the use of time series methods.

- Linear regression modelling is now widely used (e.g., Pardoe 2006) where a variable to be forecast is modelled as linear combination of potential input variables:

$$
y_{t}=\sum_{j=1}^{J} c_{j} x_{j, t}+e_{t},
$$

where $e_{t}$ denotes an iid error term with zero mean. An interesting application of regression model to forecasting is given by Byron \& Ashenfelter (1995) who use a simple regression model to predict the quality of a Grange wine using simple weather variables. However, it is far more common for regression modelling to be used to explain historical variation than for it to be used for forecasting purposes.

- In some domains, the use of nonparametric additive models for forecasting is growing (e.g., Hyndman \& Fan 2009). Here, the model is often of the form

$$
y_{t}=\sum_{j=1}^{J} f_{j}\left(x_{j, t}\right)+e_{t},
$$

where $f_{j}$ is a smooth nonlinear function to be estimated nonparametrically.

- In advertising, there is a well-developed culture of using distributed lag regression models (e.g., Hanssens et al.|2001) such as

$$
y_{t}=\sum_{j=1}^{J} \alpha \lambda^{j} x_{t-j}+e_{t},
$$

where $x_{t}$ denotes advertising expenditure in month $t, 0<\lambda<1$ and $\alpha>0$.

\subsection{Data mining methods for business forecasting}

Outside of traditional statistical modelling, an enormous amount of forecasting is done using data mining methods. Most of these methods have no formal statistical model, prediction intervals are not computed, and there is limited model checking. But some of the data-mining methods have proven powerful predictors in some contexts, especially when there is a vast quantity of available data. Predictive methods include neural networks, support vector machines and regression trees. Many of the most best-known business predictive algorithms are based on data-mining methods including the prediction of Netflix ratings and recommended books on Amazon.

\section{References}

Brown, R. G. (1959), Statistical forecasting for inventory control, McGraw-Hill, New York.

Byron, R. P. \& Ashenfelter, O. (1995), 'Predicting the quality of an unborn Grange', The Economic Record 71(212), 40-53.

Chatfield, C., Koehler, A. B., Ord, J. K. \& Snyder, R. D. (2001), 'A new look at models for exponential smoothing', Journal of the Royal Statistical Society, Series D: The Statistician 50(2), 147-159. 
Hanssens, D. M., Parsons, L. J. \& Schultz, R. L. (2001), Market Response Models: Econometric and Time Series Analysis, second edn, Kluwer Academic Publishers, Boston.

Holt, C. C. (1957), Forecasting trends and seasonals by exponentially weighted averages, O.N.R. Memorandum 52/1957, Carnegie Institute of Technology. Reprinted in International Journal of Forecasting (2004), 20, 5-10.

Hyndman, R. J. \& Fan, S. (2009), Density forecasting for long-term peak electricity demand. IEEE Transactions on Power Systems. To appear.

Hyndman, R. J., Koehler, A. B., Ord, J. K. \& Snyder, R. D. (2008), Forecasting with exponential smoothing: the state space approach, Springer-Verlag, Berlin.

Makridakis, S., Wheelwright, S. C. \& Hyndman, R. J. (1998), Forecasting: methods and applications, 3rd edn, John Wiley \& Sons, New York.

Pardoe, I. (2006), Applied regression modeling: a business approach, Wiley, Hoboken, NJ. 